\title{
A Novel Hybrid Method for Enhancement in Spectral Reflectance Recovery
}

\author{
Delibaşoğlu İbrahim, İskurt Ali, and Çetin Müfit
}

\begin{abstract}
The color information and its recovery are crucial for both hyperspectral and multispectral image analysis applications such as classification, segmentation and feature extraction. Illumination definitely affects the measured color of the objects in these images and it results in performance loss at computer vision applications. Therefore, various color constancy algorithms are applied as a fundamental preprocessing step to recover the ground truth spectral reflectance. This paper presents low-level pixel statistic based color constancy algorithms and offers a new hybrid technique for multispectral images. The proposed hybrid method is evaluated on the scenes under both simulated and real illumination. Experiments show that the proposed technique improves the performance and produces $3 \%$ and $13 \%$ less angular errors for two common datasets compared to other combinational methods.
\end{abstract}

Index Terms-Color Constancy, Multispectral, Reflectance Recovery, Illumination

\section{INTRODUCTION}

The color information is important in many computer vision and image processing applications like human-computer interaction [1] and color feature extraction [2]. The colors of image provide information about the characteristics of objects and the color of the light source. Also, the light source that provides illumination has a significant effect on the color of objects. Thus, the images of the same object taken with the same camera under different illumination sources may vary in its measured color values. The human visual system can detect these differences and recognize the colors of objects under different lighting conditions. However, this natural tendency to correct the effect of the light source in the human visual system is not fully understood [3].

The color of illumination source may cause performance loss in various computer vision applications because of the light source effects the measured color of the objects. Thus, it is important to recover the true spectral reflectance by eliminating the effects of the light source [4].

In literature several studies shows that it can be critical to eliminate the effect of light source for applications such as object detection, segmentation [5] and recognition [6]. This

Manuscript received April 20, 2016.

D. İ. Author is with Yalova University, Yalova, 77200 TURKEY

İ. A. Author is with Yalova University, Yalova, 77200 TURKEY

Ç. M. Author is with Yalova University, Yalova, 77200 TURKEY problem is called as spectral reflectance recovery or color constancy. In literature, many studies to recover spectral reflectance have been applied for RGB images [7, 8]. The state-of-the-art approaches can be divided into three types of algorithms: 1) low-level pixel statistic based, 2) gamut based and 3) learning based methods. Gamut based methods are widely used for color constancy and can also be accepted as a learning based method.

In this study, color constancy algorithms based on the pixel statistics are investigated. This is called low-level processing since processing is done at pixel (lowest) level. Retinex is one of the first studies about color constancy developed by McCann and Land [9] and many other models have been developed based on their assumption that the illumination transition is smooth. Methods for color constancy usually have been tested for RGB images including three bands. However, multispectral imaging with narrower bands has been used widely for computer vision applications in the last decade, and reflectance recovery of these narrow bands is also important to improve performance of the algorithms based on the color information. The prevalent color constancy algorithms have been summarized, implemented and tested for multispectral images. Then, a hybrid technique combining the advantageous aspects of two efficient algorithms has been proposed and tested with publicly available multispectral data sets.

\section{COLOR CONSTANCY}

The pixel values of image (I) for a diffusely reflective surface depend on light source $e(\lambda)$, surface reflectance $S(x, y, \lambda)$ and camera sensitivity function $\mathrm{C}(\lambda)$. The intensity of a pixel is modeled in (1), where $\lambda$ represents the wavelength of the light and $(\mathrm{x}, \mathrm{y})$ are the spatial coordinates.

$$
I(x, y)=\int e(\lambda) S(\mathrm{x}, \mathrm{y}, \lambda) C(\lambda) d \lambda
$$

$\mathrm{C}(\lambda)$ and $\mathrm{e}(\lambda)$ are generally unknown whereas image pixel values are known. Therefore, color constancy is an under constrained problem and the color of the light source can only be estimated under some assumptions. For instance, White-patch retinex theory based algorithm is one of the first color constancy algorithms. It works under the assumption that maximum reflectance in image channels is caused by a white patch [9]. The color channels are evaluated separately with this assumption. Maximum response for each channel is determined, and the process is also denominated as max-RGB. However, white-patch method does not guarantee that the maximum color values of the separate channels exist on the same spatial coordinates. 


$$
\max I(x, y)=\left(\max \lambda_{1}(\mathrm{x}, \mathrm{y}) \ldots \max \lambda_{\mathrm{n}}(\mathrm{x}, \mathrm{y})\right)
$$

Another well known simple color constancy algorithm is based on grey-world approach [10]. It assumes that the average reflectance in the scene is achromatic. In this assumption, it is considered that any deviation from achromaticity in the average scene color is caused by the effects of the light source. According to this method, the color of the light source can be estimated by computing the average color in the image where $\mathrm{k}$ is a normalization factor used to get the estimated illuminant spectrum in unit lengths.

$$
\frac{\int I(x, y, \lambda) d x \mathrm{dy}}{\int d x \mathrm{dy}}=k L
$$

The analysis of white-patch and grey-world algorithms shows that both algorithms can be represented as a more general minkowski norm [11].

$$
\left(\frac{\int\left(I(x, y)^{p} d x d y\right)}{\int d x \mathrm{dy}}\right)^{\frac{1}{p}}=k L
$$

Evaluating the minkowski norm for $p=1$ is equivalent to compute the average of image (I) and this equals to the grey world algorithm. When $p=\infty$, it equals to the white patch algorithm. The method based on the minkowski norm is called as shades of grey algorithm. When the performance of illumination estimation is investigated as a function of the minkowski norm as in [11], it is found that the best results are obtained when $\mathrm{p}$ equals to 6 .

The previous color constancy methods were based on the distribution of colors in the image. Grey-edge method that is similar to grey-world approach is also used an alternative approach where the incorporation of higher order image derivatives is utilized [12].

$$
\frac{\int \nabla^{n} I(x, y, \lambda) d x d y}{\int d x d y}=k L
$$

Grey-edge hypothesis assumes that the average of reflectance differences in a scene is achromatic as in the grey-world algorithm. The grey-edge algorithm can also be adapted to incorporate the minkowski norm.

Image filtering is also an option at color constancy algorithms. Gaussian filter is generally applied in order to smooth the image. For instance, general grey world algorithm applies smoothing to the image and it is considered as a different algorithm in the literature. These low-level pixel statistic based algorithms can be represented as different instantiations of the parameters in (6).

$$
\hat{L}(\lambda: n, p, \sigma)=\frac{1}{k} \iint\left\|\nabla^{n} I_{\sigma}(x, y)\right\|_{p} d x d y
$$

In this generalized formula, $n$ is the order of the differentiation, \|\|$_{p}$ is the minkowski norm and $\sigma$ is the scale of Gaussian filters. In this study, only widely accepted algorithms are summarized with different values of (n,p, $\sigma$ ) parameters. A list of these algorithms with their corresponding

\begin{tabular}{|c|c|c|c|}
\hline Method & $\mathrm{n}$ & $p$ & $\bar{\sigma} \sigma$ \\
\hline Grey World (GW) & 0 & 1 & 0 \\
\hline White Patch (WP) & 0 & $\infty$ & 0 \\
\hline Shades of Grey (SoG) & 0 & 6 & 0 \\
\hline General Grey World (GGW) & 0 & 6 & 5 \\
\hline Grey Edge $1^{\text {st }}$ order (GE1) & 1 & 1 & 6 \\
\hline Grey Edge $2^{\text {nd }}$ order (GE2) & 2 & 1 & 1 \\
\hline
\end{tabular}
parameter values is given in Table 1 .
TABLE I

COLOR CONSTANCY METHODS FOR DifFERENT INSTANTIATIONS OF PARAMETERS

After estimating the illumination spectra, a simplified linear transformation is generally used to recover the spectral reflectance $(\hat{\mathrm{I}})$;

$$
\begin{aligned}
& \hat{\mathrm{I}}(x, y, \lambda)=\operatorname{MI}(x, y, \lambda) \\
& M_{i, j}= \begin{cases}\frac{1}{L\left(\lambda_{i}\right)} & , i=j \\
0 & , \text { others }\end{cases}
\end{aligned}
$$

\section{DATASET}

\section{A. UWA Dataset}

The UWA(University of Western Australia)dataset [13] includes multi-illuminant hyperspectral scenes and is used in this study. The hyperspectral image acquisition system consists of a monochrome machine vision $\mathrm{CCD}$ camera with a resolution of $752 \times 480$ pixels. This dataset contains images of different scenes captured under five different illumination sources namely daylight, halogen, fluorescent, daylight-flourescent and halogen-flourescent. Each hyperspectral image has 33 bands between $400-720 \mathrm{~nm}$ with 10 $\mathrm{nm}$ bandwidth. RGB images for UWA dataset is shown in Fig. 1 .

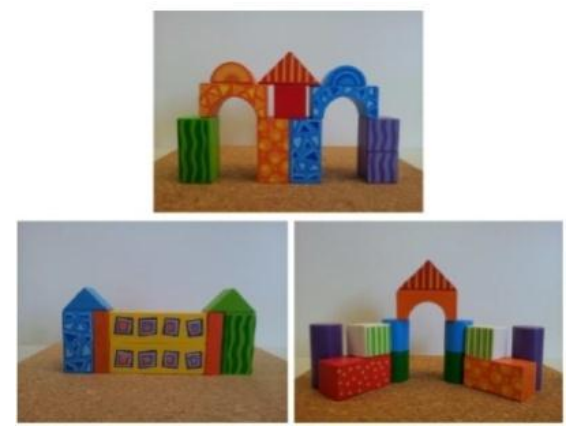

Fig. 1 UWA RGB images

\section{B. Simulated Data}

The hyperspectral images with simulated illumination are used to evaluate the color constancy algorithms. The simulated illumination is obtained from Simon Fraser University (SFU) dataset which contains spectral power distributions (SPD) of real illuminants [14]. Hyperspectral images of UWA are illuminated with SFU illumination sources and simulated dataset is prepared. Experiments on the simulated data are important since the ground truth illumination is already known while comparing with the estimated illumination. 

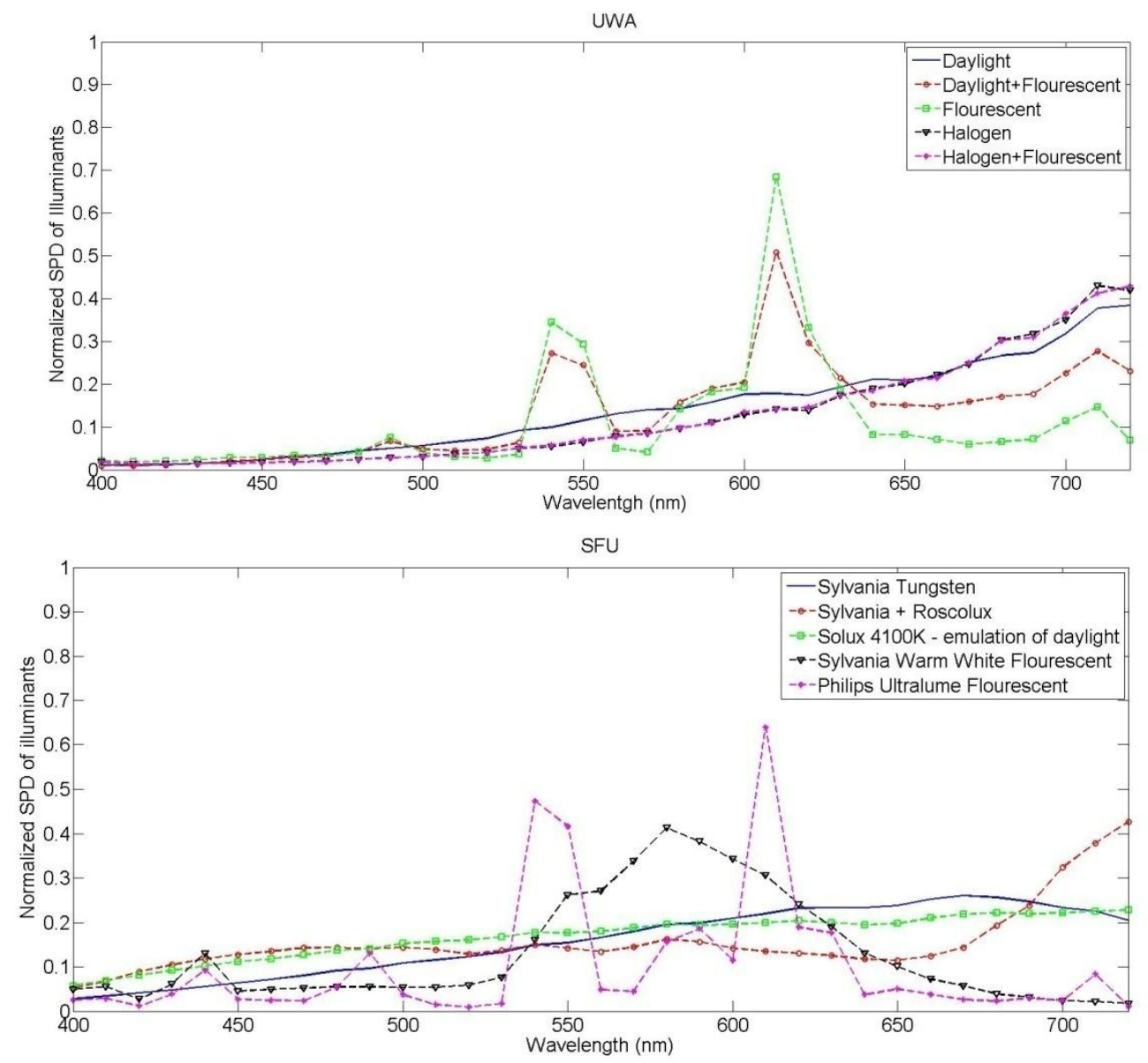

Fig. 2 Normalized SPDs of illumination sources

\section{SPDs of Illumination Sources}

In Fig.2, SPDs of all illumination sources are shown. SPDs may have considerably different characters from each other. For example, the spectral power varies very slowly between adjacent bands in the daylight illumination. This kind of illumination sources is called a smooth illuminant. On the other hand, an illumination source where the spectral power changes abruptly between certain bands is called spiky type illuminant.

\section{PROPOSED HYBRID METHOD}

The combination of different color constancy algorithms can also be applied. Looking at the algorithms in Table 1, a new approach can be suggested to see whether a combination of these different color constancy algorithms will perform better since each one has particular advantages. Averaging the whole results of the investigated algorithms could be done as a simple combination at first glance. However, such a simple combination will result in a correct estimate if and only if the majority of the algorithms individually have a good performance on that particular dataset. If some algorithms have worse performance, then it will be reasonable to exclude these. In the experiments, only two best performing algorithms (SoG and $\mathrm{GGW}$ ) are combined for a better illumination estimation.

A novel strategy is proposed in this research in order to combine these two different algorithms. In the proposed method, different algorithms are applied on different regions of wavelength-SPD graph of illumination sources. The wavelengths that have abrupt changes in SPDs of spiky illuminants are determined and the algorithm which achieves the highest accuracy in that range is applied there. The second algorithm is applied in the remaining smoother regions. For example; inFig.3, the ground truth illumination spectra and estimated illumination spectra with GGW algorithm for fluorescent type illumination is displayed. Peak values of estimated illuminant spectra for spiky type illumination source are found out and the bands with abrupt changes are determined.

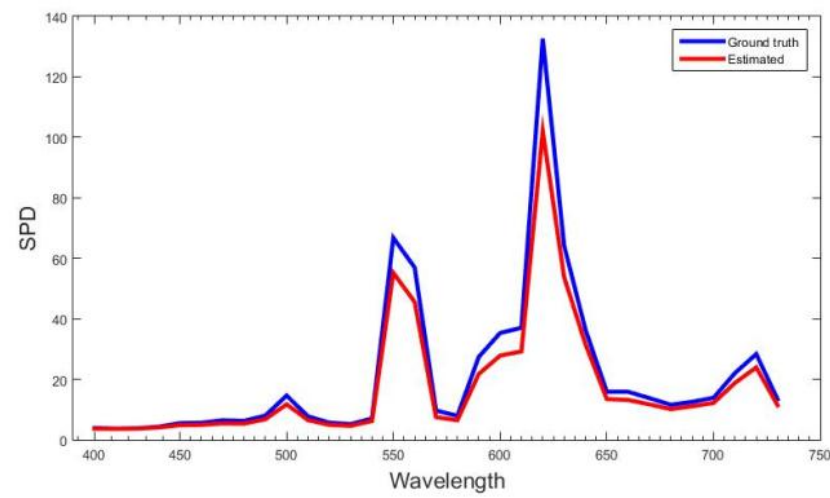

Fig. 3 UWA fluorescent illumination source ground truth and estimation values. 


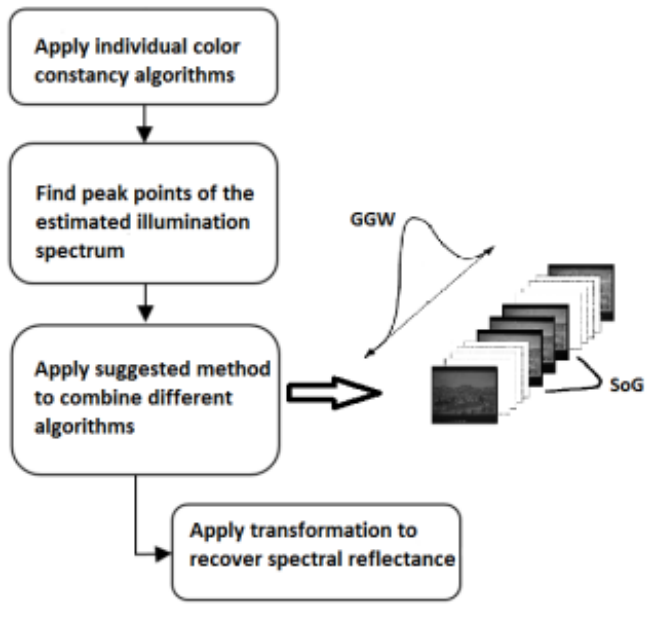

Fig. 4 Structure of proposed method

GGW method applies gauss filter for smoothing the image. Thus, it is inadequate in estimating the peak values of the illumination spectrum. By the proposed method, strong aspects of SoG and GGW algorithms that have best performance are combined. SoG and $\mathrm{gGW}$ algorithms have less angular errors respectively $3.59 \%$ and $2.98 \%$ as average for both dataset (Fig. 5). Therefore, these algorithms are chosen among others for a hybrid technique. For the cooperation of SoG and GGW algorithms; SoG algorithm is applied between $610-650 \mathrm{~nm}$ and $540-570 \mathrm{~nm}$ wavelength ranges, and GGW algorithm is applied for the rest.

\section{RESULTS}

Angular Error $(\epsilon)$ is widely used as an evaluation metric for benchmarking of color constancy algorithms [15]. In this metric, the angle between two vectors is calculated and a lower angle indicates that these vectors are close to each other. In our experiments, angular error is used as a similarity measurement and measures the angle between true illuminant spectra $(L)$ and theestimated one $(\hat{L})$.

$$
\in=\arccos \left(\frac{L}{\| L}\right)
$$

Angular errors of all individual color constancy algorithms are compared in Fig. 5 for both datasets. It is observed that overall performances of all individual algorithms are better for UWA dataset against SFU illuminated dataset according to angular error metric. In particular, SoG and GGW algorithms have the lowest angular errors and exhibit comparable performances with slight variation. Analysis of the edge based color constancy algorithms GE1 and GE2 indicates poor performance against the other algorithms.

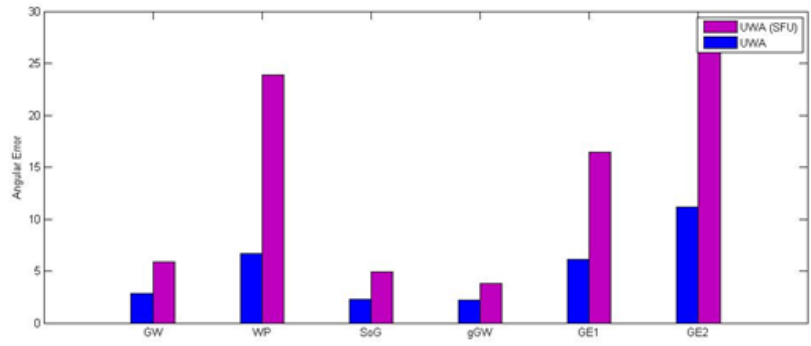

Fig. 5 Individual algorithm performances for both dataset
Proposed SoG-GGW $(*)$ hybrid method and GW-SoG, GW-GGW, SoG-GGW, GGW-GE1 methods are compared in Fig. 6. It is clear that performances of combinational methods are close to each other and that makes these methods more robust. SoG-GGW has the best performance within all combinational methods (Fig. 6) and it is observed that any other combinational methods can't exceed performances of combined-individual algorithms. Thus, the proposed hybrid method is used in order to combine these two best performing algorithms and is compared with other simple combinational methods in Fig. 6.
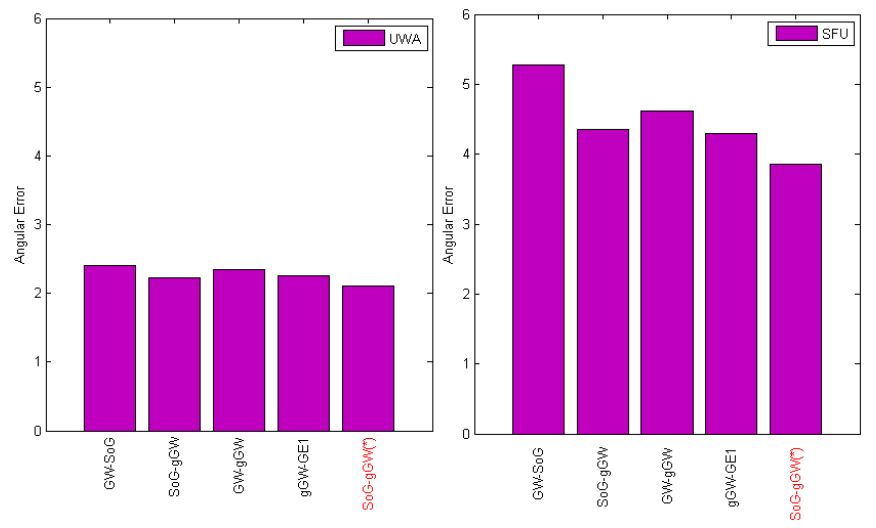

Fig. 6 Performances of combinational color constancy algorithms

SoG-GGW $(*)$ method indicates the combination of SoG and GGW algorithms with proposed hybrid method. GGW method applies gauss filter for smoothing the image, thus it is weaker to estimate peak values of the illumination spectrum. By the proposed method, the illumination source is estimated better by using SoG algorithm at peak points (wavelengths). It is observed that SoG-GGW(*) hybrid method improves the performance and gets $3 \%$ and $13 \%$ less angular errors for UWA and SFU datasets respectively.

Although combinational methods are more stable and reliable, they can't exceed the performances of individual algorithms. Fig. 7 shows ground truth and illuminated images of a scene from UWA dataset. RGB images of recovered spectral reflectance and corresponding angular errors are also shown below the images. The best performing algorithm (GGW) and the worst performing algorithm (WP) are chosen only and presented in Fig. 7 at the second row. Moreover, the proposed hybrid GGW-SoG is compared with simple GGW-SoG and other simple combinational algorithms. However, only one couple of compared images is shown at the last row of Fig. 7. It seems that only the proposed hybrid method exceeds the individual performance of GGW algorithm and other simple combinational methods are inadequate on exceeding this best individual performance. But in Fig. 7 converted RGB images does not show noticeable difference in comparison of combinational method for human perception.

\section{CONCLUSION}

A novel hybrid approach is put forward in order to combine color constancy algorithms that can be useful for multispectral images. In the proposed method, the peak values of SPDs of spiky illuminants are used to combine two different algorithms. 
If the type of the illumination source can be estimated truly and peak values of SPDs of illuminant spectra can be determined, the hybrid method improves the performances of all combinational methods and best individual performances. Consequently, the proposed hybrid method gathers strong aspects of different color constancy algorithms.

As a future search, a learning based approach can be applied to detect the type of illumination source. A set of illumination sources labeled as spiky or smooth can be used to train any artificial intelligence method and select peak regions for applying SoG and GGW hybrid method. Thus, this process can be utilized to give an automatically start to the proposed new hybrid algorithm.

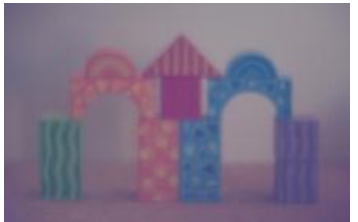

Ground Truth

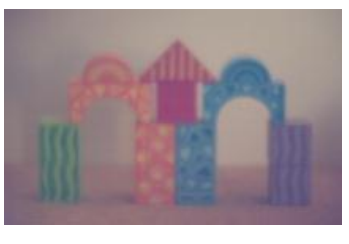

GGW

$(\epsilon=2.7871)$

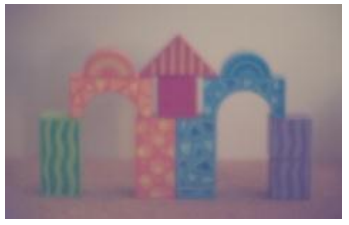

Hybrid (GGW-SoG) $(\epsilon=2.76)$

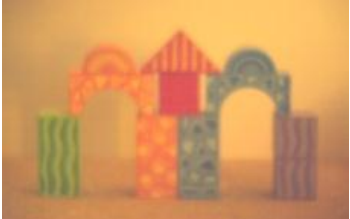

Illuminated by Philips Ultralume Flourescent

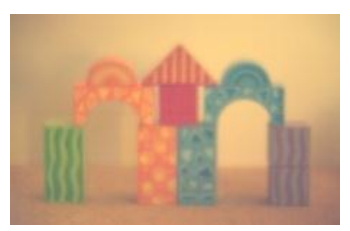

WP

$(\epsilon=3.54)$

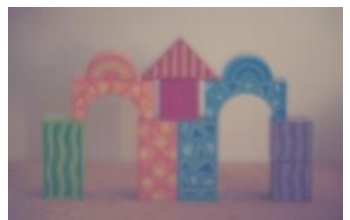

Simple Combinational (GGW-SoG) $(\in=3.1624)$
Fig. 7 Converted RGB images of recovered spectral reflectances with different color constancy algorithms and corresponding angular errors

\section{REFERENCES}

[1] Yang J., R. Stiefelhagen, U. Meier, and A. Waibel, "Visual tracking for multimodal human computer interaction," in Proc. SIGCHI Conf. Human Factors Comput. Syst, pp. 140-147,1998. http://dx.doi.org/10.1145/274644.274666

[2] Gevers T. and A. Smeulders, "Pictoseek: Combining color and shape invariant features for image retrieval," IEEE Trans. Image Process., vol. 9, no. 1, pp. 102-119, Jan. 2000.

http://dx.doi.org/10.1109/83.817602

[3] Brainard D., J. Kraft, and P. Longere, "Color constancy: Developing empirical tests of computational models," in Colour Perception: From Light to Object, R. Mausfeld and D. Heyer, Eds. London, U.K.: Oxford Univ. Press, pp. 307-334,2003.

[4] Katrasnik J., F. Pernus, and B. Likar, „A method for characterizing illumination systems for hyperspectral imaginge", Optics Express 21, no. 4, 4841-4853,2013.

[5] Brill M. H., Image segmentation by object color: A unifying framework and connection to color constancyee, JOSA A 7, no. 10, 2041-2047,1990. http://dx.doi.org/10.1364/JOSAA.7.002041

[6] Healey G. and D. Slater, „Global color constancy: Recognition of objects by use of illumination-invariant properties of color distributions"e, JOSA A 11 , no. 11, 3003-3010,1994.
http://dx.doi.org/10.1364/JOSAA.11.003003

[7] Forsyth D.A., "A novel algorithm for color constancy," International Journal of Computer Vision, vol. 5, no. 1, pp. 5-35, 1990. http://dx.doi.org/10.1007/BF00056770

[8] Li B., W. Xiong, W. Hu, and O. Wu, "Evaluating combinational color constancy methods on real-world images," in Proc. Computer Vision and Pattern Recognition, pp. 1929-1936,2011.

[9] Land E. H., "The retinex theory of color vision," Scientific American, vol. 237, no. 6, pp. 108-120, 122-123, 126, 128, 1977.

[10] Buchsbaum G., "A spatialprocessor model forobjectcolourperception," $J$. Franklin Inst., vol. FI-310, no. 1, pp. 1-26, Jul. 1980. http://dx.doi.org/10.1016/0016-0032(80)90058-7

[11] Finlayson G. and E. Trezzi, "Shades of gray and colour constancy," in IS\&T/SID Twelfth Color Imaging Conference, pp. 37-41,2004.

[12] Van De Weijer J., T. Gevers, and A. Gijsenij, "Edgebasedcolorconstancy," IEEE Transactions on ImageProcessing, vol. 16, no. 9, pp. 2207-2214, 2007. http://dx.doi.org/10.1109/TIP.2007.901808

[13] Khan, Zohaib, Faisal Shafait, and AjmalMian. "Adaptive spectral reflectance recovery using spatio-spectral support from hyperspectral images." Image Processing (ICIP), 2014 IEEE International Conference on. IEEE, 2014.

[14] Barnard, Kobus, et al. "A data set for color research." Color Research \&Application 27.3: 147-151,2002. http://dx.doi.org/10.1002/col.10049

[15] Gijsenij, Arjan, Theo Gevers, and Joost Van De Weijer. "Computational color constancy: Survey and experiments." Image Processing, IEEE Transactions on 20.9,2475-2489,2011.

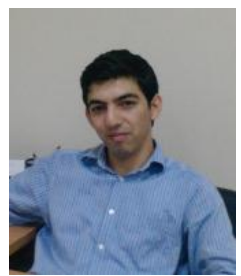

Delibaşoğlu İbrahim is research assistant in the department of Computer Engineering at Yalova University. He received his BS and MS degrees in Computer Engineering from Kocaeli University in 2009 and University of Yalova in 2013, respectively. His current research interests include image processing, remote sensing and machine learning.

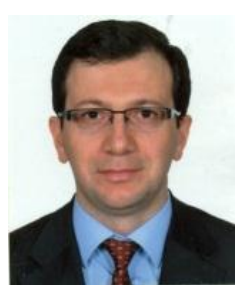

İskurt Ali is an associate professor in the department of Computer Engineering at Yalova University. He received his $\mathrm{BS}$, MS and $\mathrm{PhD}$ degrees in Electrical and Electronics Engineering from Bilkent University in 1997, Biomedical Engineering of Boğaziçi University in 2002 and Electronics and Communications Engineering of Kocaeli University in 2010, respectively. His current research interests include image processing, computer vision, pattern recognition and classification.

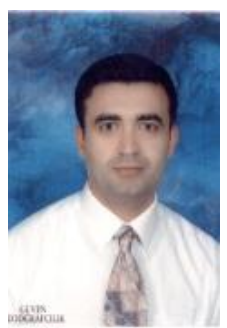

Çetin Müfit is an associate professor in the department of Computer Engineering at Yalova University. He received his $\mathrm{BS}, \mathrm{MS}$ and $\mathrm{PhD}$ degrees in Geomatics Engineering from Yildiz Technical University in 1996, Institute of Gebze Technology in 2001 and Istanbul Technical University in 2007, respectively. His current research interests include image processing and remote sensing. 